\title{
EXPERIENCES IN THE MASTOLOGY SERVICE OF A BRAZILIAN HOSPITAL DURING THE COVID-19 PANDEMIC
}

Peneloppe Santos e Lacerda', Luciana Jandre Boechat Alves', Carolina da Cunha Silveira Freitas', Saulo Bandeira Dias, Tania da Rocha Santos ${ }^{1}$

${ }^{1}$ Hospital Central da Aeronáutica, Divisão Médica, Mastologia - Rio de Janeiro (RJ), Brazil.

Introduction: The severe acute respiratory syndrome coronavirus 2 (SARS-CoV-2) has a disastrous global effect, with negative socioeconomic impacts and an unforgiven effect on health. Besides the high mortality rates and levels of sequela, the increased demand for resources caused by the pandemic also influenced the feasibility of elective procedures performed in services and hospitals worldwide. Breast cancer patients have a worse prognosis if infected with the coronavirus disease 2019 (COVID-19). However, delays in their treatment when attempting to reduce their exposure to SARSCoV-2 or delays resulted from the redirection of hospital resources toward COVID-19 efforts may similarly result in a poor prognosis. In an attempt to spare hospital resources that could be utilized in the management of COVID-19 while securing the well-being of our patients with breast cancer, we implemented a new set of guidelines. Following the recommendations from the Brazilian Society of Clinical Oncology (SBOC) and the American Society of Clinical Oncology (ASCO), we established that oncological interventions would be acceptable in situations when we could maximize patient safety. As such, in our Mastology Service of the Hospital Central da Aeronáutica (MST-HCA), a Brazilian Air Force Hospital in Rio de Janeiro, we expanded our recommendations for neoadjuvant therapies, restricted surgeries, and maintained outpatient care only for emergency cases. Objective: The aim of this study was to focus on managing the quality of care and assessing the ways to ensure adequate therapy for cancer patients during the COVID-19 pandemic. Methodology: We performed a retrospective analysis of chart information reviewing the occurrence of surgical procedures and indications for neoadjuvant therapies for patients with breast cancer from MST-HCA. The study period was between March 16 and August 1, 2020, compared with the same period in the year 2019, prior to the COVID-19 pandemic. Results: From March 16 to August 1, 2020, the MST-HCA performed a total of 20 surgeries, out of which 16 were oncological surgeries. In the same interval in 2019, there were 28 surgeries, out of which 19 were oncological surgeries. We found a $28.6 \%$ reduction in the number of surgeries during the pandemic compared with the same period in 2019. In contrast, there was an increase of $133 \%$ in the prescriptions of neoadjuvant therapies, with three patients undergoing such treatments in 2019 versus seven patients in 2020. Six of these patients had an indication for conservative surgery and only one for total mastectomy, and methods of marking the lesion were used. These methods include the placement of a metal clip in patients with an impalpable disease by the Radiology team and the performance of a skin tattoo with ink in patients with a palpable disease by the Mastology team at the outpatient clinic. Conclusions: There was a difference in the number of medical procedures due to a higher risk of SARS-COV2 infection in patients. As this is a retrospective study, we did not assess the prognosis; however, we were able to maintain the team quality standard expected even in the pandemic scenario. Using the marking methods, we were able to perform a conservative surgical approach in a safer moment for hospitalization, since the sum of senility, multiple morbidities, and cancer diagnosis constitutes a high risk of coinfection by COVID-19. 